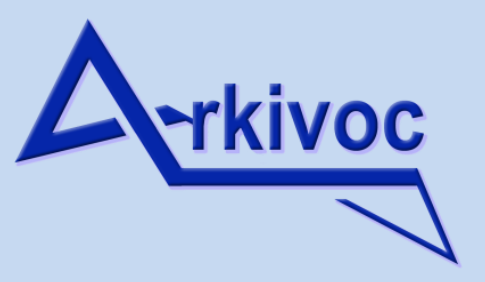

Free to Authors and Readers
A Platinum Open Access Journal for Organic Chemistry

DOAJ Seal

Arkivoc 2022, part iv, 58-69

\title{
Mannich reactions of activated 4,6-dimethoxyindoles
}

\author{
Bambang Purwono, Naresh Kumar, and David StC. Black* \\ School of Chemistry, UNSW Sydney, Sydney, NSW 2052, Australia
}

Email: d.black@unsw.edu.au

Received 10-14-2021

Accepted 11-18-2021

Published on line $12-10-2021$

\section{Abstract}

Mannich reactions of 4,6-dimethoxy-2,3-disubstituted indoles with bis(aminol)ethers catalysed by trifluoroacetic anhydride give pyrroloquinazoline derivatives through reaction at C7 and N1. 3-(4Chlorophenyl)-4,6-dimethoxyindoles with electron withdrawing substituents at C7 react with formaldehyde and dimethylamine to give the corresponding 2-dimethylaminomethyl derivatives. They also react with $N, N$ bis(methoxymethyl)benzylamine catalysed by trichloromethylsilane to give Mannich base C2-linked diindole compounds. More strongly acidic conditions (e.g. trifluoroacetic anhydride) favour the formation of 2,2'diindolylmethanes, which are preferably generated from the indoles by reaction with formaldehyde in methanolic hydrochloric acid. Reactions of a Mannich base C2-linked diindole 7,7'-dicarbaldehyde with 1,2diaminobenzene and 1,2-diaminoethane give macrocyclic diimines, and a nickel(II) complex of one of these is reported.
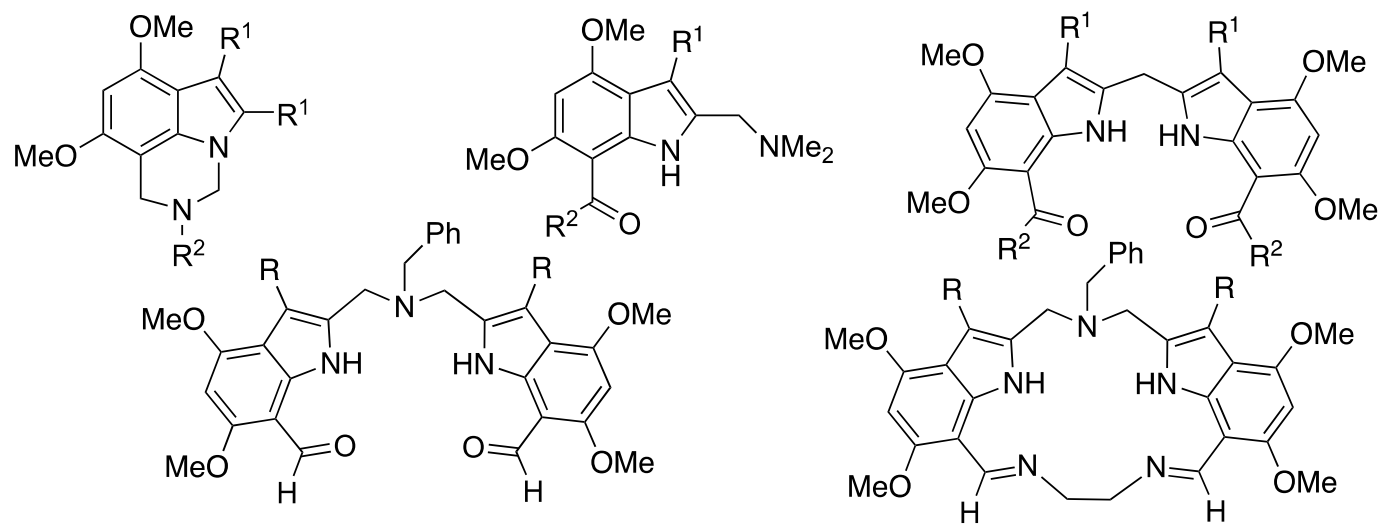

Keywords: Mannich reaction, indoles, quinazolines, imines, macrocycles, diindolylmethanes 


\section{Introduction}

The Mannich reaction has played an important role in elaborating the chemical reactivity of indoles. In this context, it was of interest to investigate the application of the Mannich reaction to activated indoles, specifically those bearing methoxy groups at $\mathrm{C} 4$ and $\mathrm{C} 6$. These electron-donating groups have the capacity to enhance not only the general reactivity of the indole ring system but also the specific activity at certain carbon and nitrogen sites. Indole 1 undergoes the classic Mannich reaction with formaldehyde and dimethylamine to give 3-(dimethylaminomethyl)indole 2 , which is also a natural product known as gramine. ${ }^{1,2}$ The application of similar reaction conditions with the activated 4,6-dimethoxyindole 3 gave the related 4,6-dimethoxygramine 4. ${ }^{3}$ 5,7-Dimethoxyindole 5 has also been reported to give the corresponding 5,7-dimethoxygramine 6 in 36\% yield. ${ }^{4}$ However, the reaction of methyl 5,7-dimethoxyindole-2-carboxylate 7 with aqueous formaldehyde and dimethylamine gave the 4-dimethylaminomethyl-substituted indole 8 in $78 \%$ yield, as the 4-position is activated by the methoxy groups and the 3-position is deactivated by the carboxylic ester at C2 (Scheme 1). ${ }^{5}$ Most of our investigations of methoxy activated indoles have been concerned with 4,6-dimethoxyindoles, and we now report the results of Mannich reactions on some examples of these compounds, with substituents at C3 or at both C2 and C3.

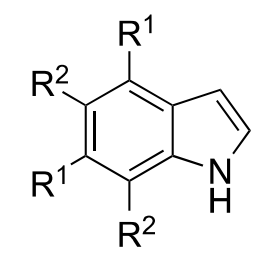

$1 \mathrm{R}^{1}=\mathrm{R}^{2}=\mathrm{H}$

$3 \mathrm{R}^{1}=\mathrm{OMe} ; \mathrm{R}^{2}=\mathrm{H}$

$5 \mathrm{R}^{1}=\mathrm{H} ; \mathrm{R}^{2}=\mathrm{OMe}$

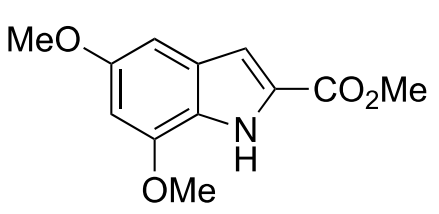

7

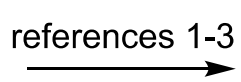

reference 4
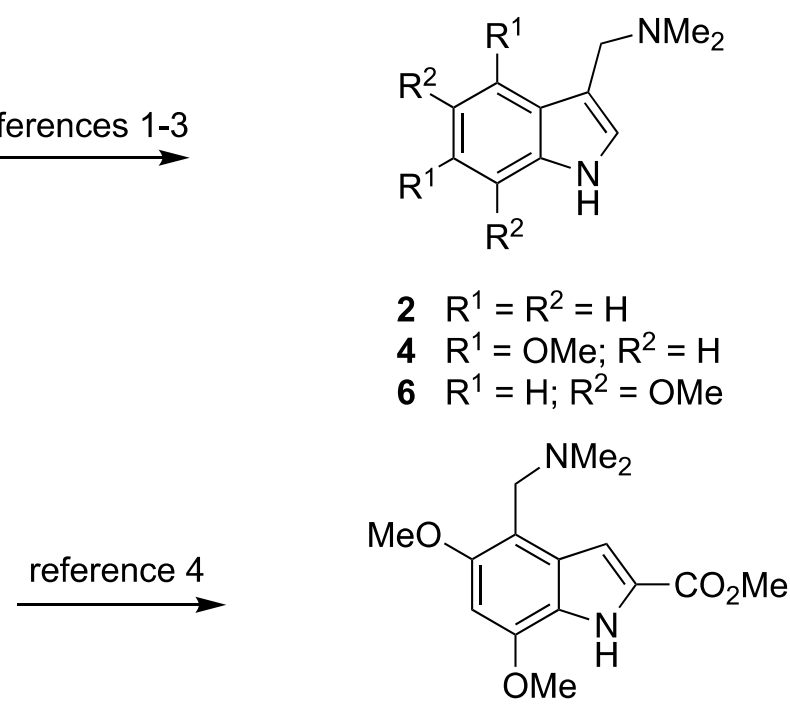

$2 \mathrm{R}^{1}=\mathrm{R}^{2}=\mathrm{H}$

$4 \mathrm{R}^{1}=\mathrm{OMe} ; \mathrm{R}^{2}=\mathrm{H}$

$6 \mathrm{R}^{1}=\mathrm{H} ; \mathrm{R}^{2}=\mathrm{OMe}$

8

Scheme 1. Previous reactions of dimethoxyindoles with formaldehyde and dimethylamine.

\section{Results and Discussion}

\section{Reactions of 2,3-disubstituted-4,6-dimethoxyindoles}

Reaction of 4,6-dimethoxy-2,3-diphenylindole 9 with formaldehyde and dimethylamine under a variety of conditions failed to yield the corresponding 7-(dimethylaminomethyl)indole 10. In general, mixtures of products were formed and the most frequently observed product was the related 7,7'-diindolylmethane $\mathbf{1 1}$ readily formed by reaction with formaldehyde alone (Scheme 2 ). ${ }^{6}$ 
<smiles>COc1cc(OC)c2c(-c3ccccc3)c(-c3ccccc3)[nH]c2c1</smiles>

9

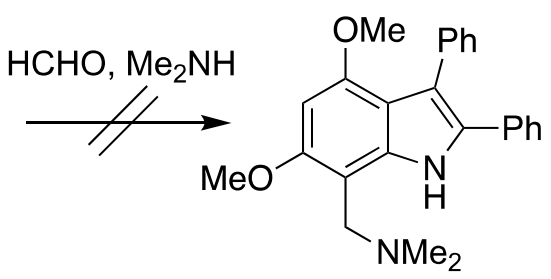

10

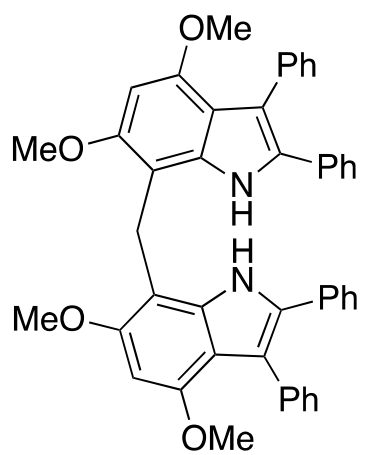

11

Scheme 2. Attempted reaction of indole 9 with formaldehyde and dimethylamine.

An important aspect of the Mannich reaction is the need for accurate optimization of reaction conditions to minimize the formation of undesired products. ${ }^{7}$ This has led to the use of preformed reagents or intermediates having the structures of methylene immonium salts or hetero-substituted aminomethyl compounds. We were attracted to the use of bis(aminol)ether derivatives which were very effectively developed by Heaney and his group, ${ }^{8-10}$ and subsequently used in natural product synthesis. ${ }^{11}$ These compounds can be preformed from primary aliphatic amines, formaldehyde and alcohols, and isolated by distillation. The possibility that these reagents might possibly lead to the formation of diindolyl amines was considered an interesting advantage. In the event, treatment of 2,3-disubstituted-4,6-dimethoxyindoles with preformed bis(aminol)ethers did not yield diindolyl amino compounds but gave rise to new cyclic compounds via a new example of an intramolecular Mannich reaction ${ }^{12}$ at both C7 and N1. Indole 9 underwent reaction with $N, N$-bis(methoxymethyl)benzylamine 12 or $N, N$-bis(ethoxymethyl)-t-butylamine 13 in dichloromethane containing trifluoroacetic acid to give the pyrroloquinazoline derivatives $\mathbf{1 4}$ and $\mathbf{1 5}$ respectively in yields of 71 and 35\%. The indole 2,3-diester 16 similarly combined with the benzylamine diether 12 to give the pyrroloquinazoline 17 in 60\% yield (Scheme 3). While these pyrroloquinazolines were isolated and fully characterized, they were found to be unstable in acid. For example, heating compound 14 in methanol containing hydrochloric acid resulted in isolation of the known 7,7'-diindolylmethane 11.<smiles>[R]c1[nH]c2cc(OC)cc(OC)c2c1[R]</smiles>

14

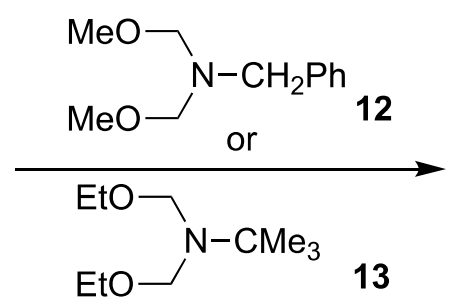

$\mathrm{CF}_{3} \mathrm{CO}_{2} \mathrm{H}, \mathrm{CH}_{2} \mathrm{Cl}_{2}$

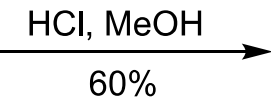<smiles>[R]c1c([R])n2c3c(c(OC)cc(OC)c13)CN([R])C2</smiles>

$14 \mathrm{R}^{1}=\mathrm{Ph} ; \mathrm{R}^{2}=\mathrm{CH}_{2} \mathrm{Ph}(71 \%)$

$15 \mathrm{R}^{1}=\mathrm{Ph} ; \mathrm{R}^{2}=\mathrm{CMe}_{3}(35 \%)$

$17 \mathrm{R}^{1}=\mathrm{CO}_{2} \mathrm{Me} ; \mathrm{R}^{2}=\mathrm{CH}_{2} \mathrm{Ph}(60 \%)$

11

Scheme 3. Formation of pyrroloquinazoline derivatives 14, 15 and 17. 


\section{Reactions of 3-(4-chlorophenyl)-4,6-dimethoxyindole 18.}

Reaction of the indole $\mathbf{1 8}$ with formaldehyde and dimethylamine under a variety of conditions gave complex product mixtures. Similar results were observed with other primary and secondary amines but there was indication of aminomethylation at the indole nitrogen atom. In general the products were too unstable to allow isolation and full characterisation. It became clear that these activated indoles were too reactive and also too unselective, so attention turned to related structures with electron-withdrawing substituents at $\mathrm{C7}$.

\section{Reactions of 3-(4-chlorophenyl)-4,6-dimethoxyindoles bearing electron-withdrawing substituents at C7.}

Various 3-(4-chlorophenyl)-4,6-dimethoxyindoles were available for investigation. Indole 19 with a 7-(4chlorobenzoyl) group, and the related 7-trichloroacetylindole 20 have been previously reported. ${ }^{13}$ The latter compound $\mathbf{2 0}$ was converted by treatment with methanol into the new indole $\mathbf{2 1}$ with a 7-methoxycarbonyl group. Indoles 19 and $\mathbf{2 1}$ reacted smoothly with formaldehyde and dimethylamine to give the related 2dimethylaminomethyl-substituted indoles $\mathbf{2 2}$ and $\mathbf{2 3}$ respectively in yields of 70 and $75 \%$ (Scheme 4).
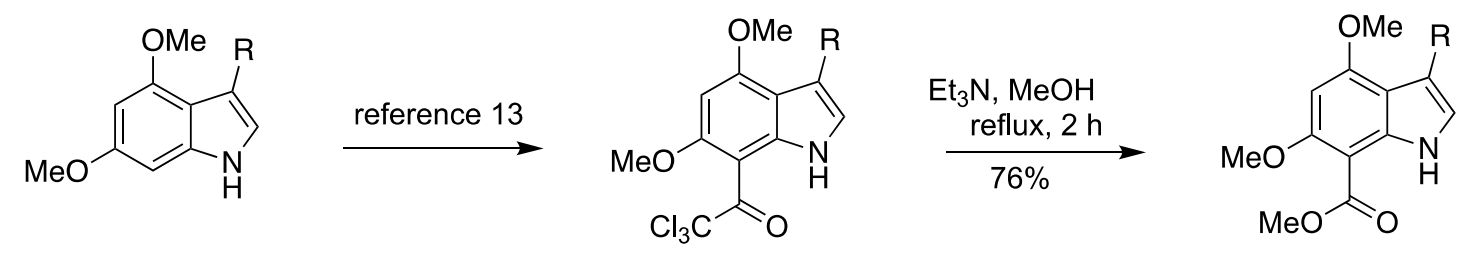

$$
18 \mathrm{R}=4-\mathrm{ClC}_{6} \mathrm{H}_{4}
$$<smiles>[R]C(=O)c1c(OC)cc(OC)c2[nH]cc([R])c12</smiles>

$19 \mathrm{R}=4-\mathrm{ClC}_{6} \mathrm{H}_{4}$<smiles>COC(=O)c1c(OC)cc(OC)c2[nH]cc(P)c12</smiles>

$21 \mathrm{R}=4-\mathrm{ClC}_{6} \mathrm{H}_{4}$

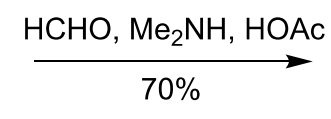

$20 \mathrm{R}=4-\mathrm{ClC}_{6} \mathrm{H}_{4}$<smiles>[R]C(=O)c1c(OC)cc(OC)c2[nH]c(CN(C)C)c([R])c12</smiles>

$22 \mathrm{R}=4-\mathrm{ClC}_{6} \mathrm{H}_{4}$

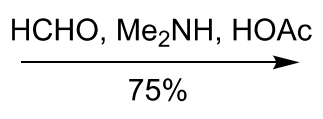

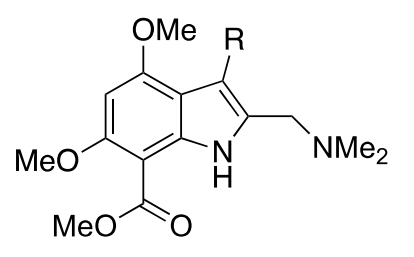

$23 \mathrm{R}=4-\mathrm{ClC}_{6} \mathrm{H}_{4}$

Scheme 4. Formation of 2-aminomethylindoles 22 and 23.

The 7-trichloroacetylindole 20 combined with $N, N$-bis(methoxymethyl)- benzylamine $\mathbf{1 2}$ in a 2:1 ratio in dichloromethane containing either trifluoroacetic anhydride or preferably trichloromethylsilane to give the Mannich base linked diindole compound 24 in 60 and 64\% yields respectively. In the case of a similar reaction of the known 7-formylindole $\mathbf{2 5} \mathbf{5}^{14}$ with amine diether $\mathbf{1 2}$ and trichloromethylsilane, the related Mannich base linked diindolyl compound $\mathbf{2 6}$ was obtained in $\mathbf{8 0 \%}$ yield (Scheme 5 ). 
<smiles>[R]C(=O)c1c(OC)cc(OC)c2[nH]cc([R])c12</smiles>

12, $\mathrm{MeSiCl}_{3}, \mathrm{CH}_{2} \mathrm{Cl}_{2}$ rt, $2 \mathrm{~d}$
$20 \mathrm{R}^{1}=4-\mathrm{ClC}_{6} \mathrm{H}_{4} ; \mathrm{R}^{2}=\mathrm{CCl}_{3}$

$25 \mathrm{R}^{1}=4-\mathrm{ClC}_{6} \mathrm{H}_{4} ; \mathrm{R}^{2}=\mathrm{H}$<smiles>[R]C(=O)c1c(OC)cc(OC)c2c([R])c(CN(Cc3ccccc3)Cc3[nH]c4c([R])c(OC)cc(OC)c4c3[R])[nH]c12</smiles>

$24 \mathrm{R}^{1}=4-\mathrm{ClC}_{6} \mathrm{H}_{4} ; \mathrm{R}^{2}=\mathrm{CCl}_{3}(64 \%)$

$26 \mathrm{R}^{1}=4-\mathrm{ClC}_{6} \mathrm{H}_{4} ; \mathrm{R}^{2}=\mathrm{H}(80 \%)$

Scheme 5. Formation of diindolyl Mannich bases 24 and 26.

However, the acid catalyst is quite crucial, because the use of trifluoroacetic anhydride in the case of indoles $\mathbf{2 1}$ and $\mathbf{2 5}$ did not give the Mannich base linked products but instead gave the 2,2' -diindolylmethanes $\mathbf{2 7}$ and $\mathbf{2 8 ^ { 1 5 }}$ respectively (Scheme 6). The milder catalytic conditions using trichloromethylsilane are required for isolation of the Mannich base, and even under these conditions some diindolylmethane is usually also observed. In reactions catalyzed by trifluoroacetic anhydride, good yields of the diindolylmethanes can be obtained, but the optimal route to these compounds is via reaction of the indoles with formaldehyde alone in methanolic hydrochloric acid. In this way, the indoles 19, 20, 21, and 25 were converted into the diindolylmethanes 27-30. The diindolylmethane diamide $\mathbf{3 1}$ was prepared from compound 30, by treatment with ammonia, and the diindolyl diacid $\mathbf{3 2}$ by hydrolysis of the diester $\mathbf{2 7}$ (Scheme 6).<smiles>[R]C(=O)c1c(OC)cc(OC)c2[nH]cc([R])c12</smiles>

$\mathrm{HCHO}, \mathrm{HCl}, \mathrm{MeOH}$
reflux

$$
\begin{array}{ll}
21 & \mathrm{R}^{1}=4-\mathrm{CIC}_{6} \mathrm{H}_{4} ; \mathrm{R}^{2}=\mathrm{OMe} \\
25 & \mathrm{R}^{1}=4-\mathrm{ClC}_{6} \mathrm{H}_{4} ; \mathrm{R}^{2}=\mathrm{H} \\
19 & \mathrm{R}^{1}=\mathrm{R}^{2}=4-\mathrm{ClC}_{6} \mathrm{H}_{4} \\
20 & \mathrm{R}^{1}=4-\mathrm{CIC}_{6} \mathrm{H}_{4} ; \mathrm{R}^{2}=\mathrm{CCl}_{3}
\end{array}
$$<smiles>[R]c1c(Cc2[nH]c3c(C(=O)C(Cl)(Cl)Cl)c(OC)cc(OC)c3c2[R])[nH]c2c(C(=O)C(Cl)(Cl)Cl)c(OC)cc(OC)c12</smiles>

$30 \mathrm{R}=4-\mathrm{ClC}_{6} \mathrm{H}_{4}$<smiles>[R]c1c(Cc2[nH]c3c(C(=O)OC)cc(OC)c(C)c3c2[R])[nH]c2c(C(=O)OC)c(OC)cc(OC)c12</smiles>

$27 \mathrm{R}=4-\mathrm{ClC}_{6} \mathrm{H}_{4}$

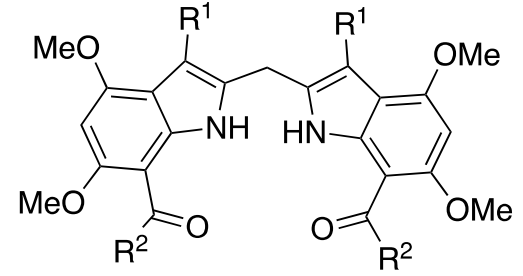

$27 \mathrm{R}^{1}=4-\mathrm{ClC}_{6} \mathrm{H}_{4} ; \mathrm{R}^{2}=\mathrm{OMe}(56 \%)$

$28 \mathrm{R}^{1}=4-\mathrm{ClC}_{6} \mathrm{H}_{4} ; \mathrm{R}^{2}=\mathrm{H}$ (reference 12)

$29 \mathrm{R}^{1}=\mathrm{R}^{2}=4-\mathrm{ClC}_{6} \mathrm{H}_{4}(70 \%)$

$30 \mathrm{R}^{1}=4-\mathrm{ClC}_{6} \mathrm{H}_{4} ; \mathrm{R}^{2}=\mathrm{CCl}_{3}(68 \%)$

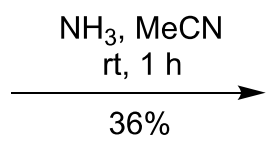

$31 \mathrm{R}=4-\mathrm{ClC}_{6} \mathrm{H}_{4}$<smiles>[R]c1c(Cc2[nH]c3c(C(=O)O)c(OC)cc(OC)c3c2[R])[nH]c2c(C(=O)O)c(OC)cc(OC)c12</smiles>

$32 \mathrm{R}=4-\mathrm{ClC}_{6} \mathrm{H}_{4}$

Scheme 6. Formation of diindolylmethanes 27- 32. 
The Mannich base linked diindolyl compound 26 underwent reactions with 1,2-diaminobenzene and 1,2-diaminoethane in boiling toluene to give the macrocyclic diimines $\mathbf{3 3}$ and $\mathbf{3 4}$ respectively in yields of 40 and $30 \%$. Reasonable to excellent yields of macrocyclic imines derived from indole-7-aldehydes have been reported, without the need for high dilution, possibly as a result of strong hydrogen bonding between the imine $\mathrm{N}$ and the indole $\mathrm{NH} .{ }^{15-17}$ The macrocyclic diimine $\mathbf{3 3}$ also gave a characterisable nickel(II) complex 35, which shows four coordinate square planar geometry with no additional electron donation from the bridging nitrogen lone pair (Scheme 7).<smiles>[R]c1c(OC)cc(OC)c2c([R])c(CN(Cc3ccccc3)Cc3[nH]c4c(C=O)cc(OC)c(OC)c4c3[R])[nH]c12</smiles>

$28 \mathrm{R}=4-\mathrm{ClC}_{6} \mathrm{H}_{4}$

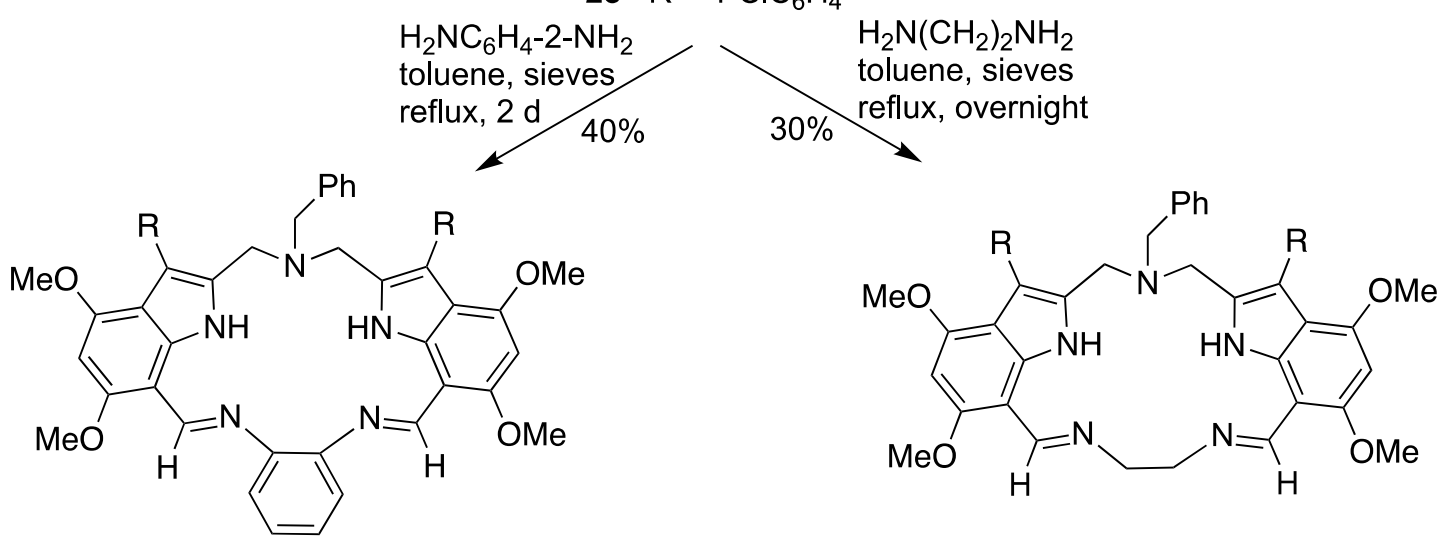

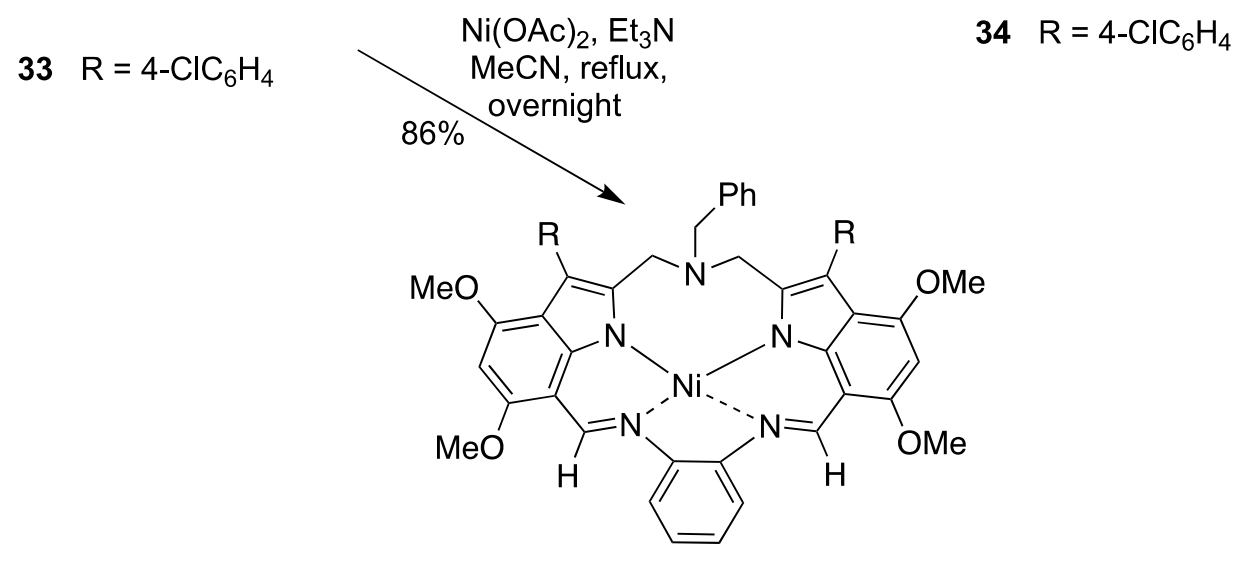

$35 \mathrm{R}=4-\mathrm{ClC}_{6} \mathrm{H}_{4}$

Scheme 7. Formation of macrocyclic diimines 33 and 34.

\section{Conclusions}

4,6-Dimethoxyindoles show enhanced nucleophilic reactivity and this can become problematic for their Mannich reactions. However, the presence of stabilising aryl groups, and especially electron-withdrawing 
substituents, enables clean reactions in good yields. The use of preformed Mannich reagents such as bis(aminol)ethers in mildly acidic conditions gives Mannich base linked diindolyl compounds. Given careful control, the Mannich reaction methodology offers useful access to a range of new structures.

\section{Experimental Section}

General. ${ }^{1} \mathrm{H}$ and ${ }^{13} \mathrm{C}$ NMR spectra were recorded on a Bruker $\mathrm{AC} 300 \mathrm{~F}\left({ }^{1} \mathrm{H}: 300 \mathrm{MHz},{ }^{13} \mathrm{C}: 75.5 \mathrm{MHz}\right)$ or a Bruker AM500 spectrometer. The chemical shifts $(\delta)$ and coupling constants $(J)$ are expressed in ppm and hertz respectively. Carbon attribution $\mathrm{C}, \mathrm{CH}, \mathrm{CH}_{2}$ and $\mathrm{CH}_{3}$ were determined by ${ }^{13} \mathrm{C}$, DEPT and HMQC experiments. Infrared (IR) spectra were recorded on a Mattson Genesis Series FTIR spectrometer using potassium bromide disks, except where specified. Ultraviolet and visible (UV/Vis) spectra were recorded in tetrahydrofuran, methanol or chloroform using a Carey 100 spectrometer. Mass spectra were recorded on a VG Quattro MS (EI) or a Finnigan MAT (MALDI). High resolution mass spectrometry (HRMS) was carried out at the Research School of Chemistry, Australian National University. Melting points were measured using a Mel-Temp melting point apparatus. Microanalyses were performed at the UNSW Microanalytical Unit and at the Campbell Microanalytical Laboratory, University of Otago, New Zealand. Column chromatography was carried out using Merck 230-400 mesh silica gel or Merck 70-230 mesh silica gel, whilst preparative TLC was performed using Merck 60GF 254 silica gel.

5-Benzyl-5,6-dihydro-7,9-dimethoxy-1,2-diphenyl-4H-pyrrolo[3,2,1-ij]quinazoline (14). A solution of indole 9 (0.20 g, $0.5 \mathrm{mmol})$ and compound 12 (94 mg, $0.5 \mathrm{mmol})$ in dichloromethane $(10 \mathrm{~mL})$ was treated with trifluoroacetic acid $(0.48 \mathrm{mmol}, 0.02 \mathrm{~mL})$. The reaction mixture was stirred at $\mathrm{rt}$ for 30 min, water was added and the organic layer was separated and washed with sat. $\mathrm{NaHCO}_{3}$ solution. The organic layer was dried $\left(\mathrm{MgSO}_{4}\right)$ and concentrated. The residue was purified by chromatography with $\mathrm{CH}_{2} \mathrm{Cl}_{2}$ as eluent to yield compound $14(0.16 \mathrm{~g}, 71 \%)$ as a pale white solid, $\mathrm{mp} 175^{\circ} \mathrm{C}$ (from $\left.\mathrm{CH}_{2} \mathrm{Cl}_{2}-\mathrm{MeOH}\right) . \mathrm{IR}\left(\mathrm{v}_{\max }, \mathrm{cm}^{-1}\right): 1600,1500$, 1320, 1200, 1100, 1050, 740, 700. UV/Vis $\left(\lambda_{\max }, \mathrm{nm}, \varepsilon, \mathrm{cm}^{-1} \mathrm{M}^{-1}\right): 212(40,000), 248(31,000), 319(14,900) .{ }^{1} \mathrm{H}$ NMR $\left(300 \mathrm{MHz}, \mathrm{CDCl}_{3}\right): \delta_{\mathrm{H}} 3.67,4.19,4.87$ (each 2H, 3s, $\mathrm{CH}_{2}$ ), 3.78, 3.88 (each $\left.3 \mathrm{H}, 2 \mathrm{~s}, \mathrm{OMe}\right), 6.33(1 \mathrm{H}, \mathrm{s}, \mathrm{H} 8)$, 7.13-7.33 (13H, m, aryl), $7.34\left(2 \mathrm{H}, \mathrm{d}, J 7.0 \mathrm{~Hz}\right.$, aryl). ${ }^{13} \mathrm{C} \mathrm{NMR}\left(75 \mathrm{MHz}, \mathrm{CDCl}_{3}\right): \delta_{\mathrm{C}} 47.0,55.8,63.5\left(\mathrm{CH}_{2}\right), 56.9$, 57.1 (OMe), 90.3 (C8), 127.1, 127.3, 128.2, 128.4, 129.2, 130.4, 131.5, (aryl CH), 98.4, 110.4, 114.4, 125.4, 134.6, 135.0, 135.6, 138.2, 152.3, 153.5 (aryl C). MS (+EI, $m / z, \%): 461$ (M+1, 10), 460 (M, 35$), 342$ (45), 341 (85), 254 (20). Anal. calcd for $\mathrm{C}_{31} \mathrm{H}_{28} \mathrm{~N}_{2} \mathrm{O}_{2} .0 .5 \mathrm{H}_{2} \mathrm{O}$ : C, 79.3; $\mathrm{H}, 6.2 ; \mathrm{N}, 6.0$. Found: C, 79.3; $\mathrm{H}, 6.3 ; \mathrm{N}, 5.9 \%$.

Heating compound 14 (46 mg, $0.1 \mathrm{mmol})$ under reflux in methanol $(20 \mathrm{~mL})$ containing conc. $\mathrm{HCl}(1 \mathrm{~mL})$ for $3 \mathrm{~h}$ gave di-[7-(4,6-dimethoxy-3,4-diphenyl)indolyl]methane $11(20 \mathrm{mg}, 60 \%)$, identical with an authentic sample. ${ }^{6}$ 5-t-Butyl-5,6-dihydro-7,9-dimethoxy-1,2-diphenyl-4H-pyrrolo[3,2,1-ij]quinazoline (15). This was prepared according to the method of preparation of compound 14 using indole $16(0.36 \mathrm{~g}, 1.0 \mathrm{mmol})$ and compound 13 $(0.20 \mathrm{~g}, 1.0 \mathrm{mmol})$. The residue was purifed using chromatography with $\mathrm{CH}_{2} \mathrm{Cl}_{2}$ as eluent to yield compound 15 $(0.15 \mathrm{~g}, 35 \%)$ as a white solid, $\mathrm{mp} 196{ }^{\circ} \mathrm{C}$ (from $\left.\mathrm{CH}_{2} \mathrm{Cl}_{2}-\mathrm{MeOH}\right)$. IR $\left(v_{\max }, \mathrm{cm}^{-1}\right): 1600,1250,1200,1120,1070$, 1050,700. UV/Vis $\left(\lambda_{\max }, \mathrm{nm}, \varepsilon, \mathrm{cm}^{-1} \mathrm{M}^{-1}\right): 213(34,300), 319(15,300) .{ }^{1} \mathrm{H}$ NMR $\left(300 \mathrm{MHz}, \mathrm{CDCl}_{3}\right): \delta_{\mathrm{H}} 1.16(9 \mathrm{H}, \mathrm{s}$, $t$-Bu), 3.74, 3.93 (each 3H, 2s, OMe), 4.21, 4.91 (each 2H, 2s, $\left.\mathrm{CH}_{2}\right), 6.30(1 \mathrm{H}, \mathrm{s}, \mathrm{H} 8), 7.17-7.33(10 \mathrm{H}, \mathrm{m}, \operatorname{aryl}) .{ }^{13} \mathrm{C}$ NMR (75 MHz, CDCl 3 ): $\delta_{c} 27.5$ (Me), 41.6 (CMe), 54.5, $59.5\left(\mathrm{CH}_{2}\right), 55.8,57.0$ (OMe), 90.2 (C8), 125.3, 127.1, $127.4,128.3,130.5,131.4$ (aryl CH), 101.2, 110.6, 114.7, 131.8, 134.0, 135.7, 135.8, 150.8, 153.2 (aryl C). MS (+El, m/z, \%): 427 (M+1, 10), 426 (M, 30), 426 (30), 342 (50), 341 (100), 254 (20). Anal. calcd for $\mathrm{C}_{28} \mathrm{H}_{30} \mathrm{~N}_{2} \mathrm{O}_{2.0} .6$ $\mathrm{CH}_{2} \mathrm{Cl}_{2}$ : C, 71.9; $\mathrm{H}, 6.6 ; \mathrm{N}, 5.9$. Found: $\mathrm{C}, 72.0 ; \mathrm{H}, 6.8 ; \mathrm{N}, 5.9 \%$. 
Dimethyl 5-benzyl-5,6-dihydro-7,9-dimethoxy-4H-pyrrolo[3,2,1-ij]quinazoline-1,2-dicarboxylate (17). This was prepared according to the method of preparation of compound 14 using indole $16(0.20 \mathrm{~g}, 0.60 \mathrm{mmol})$ and compound $13(0.11 \mathrm{~g}, 0.60 \mathrm{mmol})$. The residue was purified by chromatography with EtOAc-light petroleum 1:1 as eluent to yield compound $17(0.15 \mathrm{~g}, 60 \%)$ as a white solid, $\mathrm{mp} 130{ }^{\circ} \mathrm{C}$ (from $\mathrm{CH}_{2} \mathrm{Cl}-\mathrm{MeOH}_{2}$ ). IR $\left(v_{\max }, \mathrm{cm}^{-1}\right): 1700,1610,1500,1200,1020,970,800,730,700$. UV/Vis $\left(\lambda_{\max }, \mathrm{nm}, \varepsilon, \mathrm{cm}^{-1} \mathrm{M}^{-1}\right): 251(28,300)$, 211 (27,300), 309 (15,200). ${ }^{1} \mathrm{H}$ NMR $\left(300 \mathrm{MHz}_{\mathrm{CDCl}}\right.$ ): $\delta_{\mathrm{H}} 3.58,4.05,5.33$ (each $2 \mathrm{H}, 3 \mathrm{~s}, \mathrm{CH}_{2}$ ), $3.82,3.84$ (each $3 \mathrm{H}, 2 \mathrm{~s}, \mathrm{COOMe}), 3.91,3.96$ (each $3 \mathrm{H}, 2 \mathrm{~s}, \mathrm{OMe}), 6.26(1 \mathrm{H}, \mathrm{s}, \mathrm{H} 8), 7.22-7.34\left(5 \mathrm{H}, \mathrm{m}\right.$, aryl). ${ }^{13} \mathrm{C} \mathrm{NMR}(75 \mathrm{MHz}$, $\left.\mathrm{CDCl}_{3}\right): \delta_{c} 45.4,51.9,65.6\left(\mathrm{CH}_{2}\right), 52.5,55.7,56.7,56.9$ (OMe), 90.3 (C8), 127.4, 128.3, $129.0(\operatorname{aryl~CH}), 98.0$, 109.0, 114.4, 122.9, 135.8, 138.0, 153.4, 154.7 (aryl C), 161.2, 167.2 (C=O). MS (+El, $m / z, \%): 425$ (M+1, 10), 424 (M, 30), 426 (30), 306 (25), 305 (40), 275 (20), 274 (75), 212 (40), 91 (100). Anal. calcd for $\mathrm{C}_{23} \mathrm{H}_{24} \mathrm{~N}_{2} \mathrm{O}_{6}: \mathrm{C}_{\text {, }}$ $65.1 ; H, 5.7 ; \mathrm{N}, 6.6$. Found: C, 65.0; $\mathrm{H}, 5.7 ; \mathrm{N}, 6.6 \%$.

Methyl 3-(4-chlorophenyl)-4,6-dimethoxyindole-7-carboxylate (21). 7-Trichloroacetylindole 20 (0.60 g, 1.40 $\mathrm{mmol})$ was partially dissolved in anhydrous $\mathrm{MeOH}(40 \mathrm{~mL})$ containing $\mathrm{Et}_{3} \mathrm{~N}(1 \mathrm{ml})$. The mixture was heated under reflux for $2 \mathrm{~h}$ and allowed to cool to $\mathrm{rt}$, whereupon a yellow precipitate formed. The solid was filtered off, washed with $\mathrm{MeOH}$ and dried to afford compound 21 (0.40 g, 76\%), mp $193{ }^{\circ} \mathrm{C}$ (from $\mathrm{CH}_{2} \mathrm{Cl}_{2}-\mathrm{MeOH}$ ). IR $\left(v_{\max }, \mathrm{cm}^{-1}\right): 3350,1660,1560,1255,1210$. UV/Vis $\left(\lambda_{\max }, \mathrm{nm}, \varepsilon, \mathrm{cm}^{-1} \mathrm{M}^{-1}\right): 241(30,800), 206(21,500), 306$ $(14,400) .{ }^{1} \mathrm{H}$ NMR $\left(300 \mathrm{MHz}, \mathrm{CDCl}_{3}\right): \delta_{\mathrm{H}} 3.89,3.97,3.99$ (each 3H, 3s, OMe), $6.28(1 \mathrm{H}, \mathrm{s}, \mathrm{H} 5), 7.10(1 \mathrm{H}, \mathrm{d}, J$ 2.5Hz, H2), 7.31-7.51 (4H, m, aryl), 10.27 (1H, br s, NH). $\left.{ }^{13} \mathrm{C} \mathrm{NMR} \mathrm{(75} \mathrm{MHz,} \mathrm{CDCl}_{3}\right):$ oc 51.7, 55.2, 55.4 (OMe), 88.9 (C5), 121.5 (2), 127.7, 130.7 (aryl CH), 95.5, 110.5, 117.5, 131.7, 134.2, 139.5, 158.8, 160.2 (aryl C), 167.8 $(\mathrm{C}=\mathrm{O})$. MS (+El, m/z, \%): $347\left(\mathrm{M}+2, \mathrm{Cl}^{37 / 37}, 17\right), 345\left(\mathrm{M}, \mathrm{Cl}^{35 / 35}, 55\right), 315$ (35), 313 (100). Anal. calcd for $\mathrm{C}_{18} \mathrm{H}_{16} \mathrm{ClNO}_{4}$. 0.4 $\mathrm{H}_{2} \mathrm{O}: \mathrm{C}, 61.3 ; \mathrm{H}, 4.8 ; \mathrm{N} 4.0$. Found: $\mathrm{C}, 61.3 ; \mathrm{H}, 4.3 ; \mathrm{N} 3.7 \%$.

7-(4-Chlorobenzoyl)-3-(4-chlorophenyl)-4,6-dimethoxy-2-( $N, N$ '-dimethyl-aminomethyl)indole (22). A cooled mixture of dimethylamine $(40 \%, 0.1 \mathrm{~mL})$ and formaldehyde $(37 \%, 0.01 \mathrm{~mL})$ in glacial AcOH $(1 \mathrm{~mL})$ was added dropwise into a solution of indole $19(85 \mathrm{mg}, 0.2 \mathrm{mmol})$ in glacial $\mathrm{AcOH}(5 \mathrm{~mL})$. The mixture was stirred at 50 ${ }^{\circ} \mathrm{C}$ overnight, then diluted with water $(20 \mathrm{~mL})$. The mixture was cooled in an ice-bath and $\mathrm{NaOH}$ pellets were added slowly until the mixture was basic. The mixture was extracted with EtOAc and the organic layer washed with water and dried $\left(\mathrm{MgSO}_{4}\right)$. The solvent was evaporated off to yield compound $22(67 \mathrm{mg}, 70 \%)$ as a white solid, $\mathrm{mp} 198^{\circ} \mathrm{C}\left(\mathrm{CH}_{2} \mathrm{Cl}_{2}\right.$-light petroleum). IR ( $\left.v_{\max } \mathrm{cm}^{-1}\right): 3420,1610,1580,1560,1340,1260,1170,1130,980$, 820, 760. UV/Vis $\left(\lambda_{\max }, \mathrm{nm}, \varepsilon, \mathrm{cm}^{-1} \mathrm{M}^{-1}\right): 206(45,600), 259(29,900), 332(11,800) .{ }^{1} \mathrm{H} \mathrm{NMR}\left(300 \mathrm{MHz} \mathrm{CDCl}_{3}\right): \delta_{\mathrm{H}}$ $2.19(6 \mathrm{H}, \mathrm{s}, \mathrm{Me}), 3.47\left(2 \mathrm{H}, \mathrm{s}, \mathrm{CH}_{2}\right), 3.62,3.81$ (each 3H, 2s, OMe), $6.17(1 \mathrm{H}, \mathrm{s}, \mathrm{H} 5), 7.33(4 \mathrm{H}, \mathrm{m}, \operatorname{aryl}), 7.37(2 \mathrm{H}$, d, J $8.7 \mathrm{~Hz}$, aryl), $7.60\left(2 \mathrm{H}, \mathrm{d}, J 8.7 \mathrm{~Hz}\right.$, aryl), $10.28\left(1 \mathrm{H}\right.$, br s, NH). ${ }^{13} \mathrm{C} \mathrm{NMR}\left(75 \mathrm{MHz}, \mathrm{CDCl}_{3}\right): \delta_{\mathrm{c}} 45.4(\mathrm{Me}), 54.4$ $\left(\mathrm{CH}_{2}\right)$, 55.3, 56.2 (OMe), 87.8 (C5), 127.4, 127.8, 129.9, 132.2 (aryl CH), 103.8, 112.1, 114.2, 131.8, 132.6, 133.7, 136.8, 137.7, 140.5, 159.2 (aryl C), 194.3 (C=0). MS (+EI, $m / z, \%): 486(M+4,7), 484(M+2,35), 482$ (M,50), 440 (45), 438 (70), 405 (20), 403 (65), 139 (90), 58 (100). Anal. calcd for $\mathrm{C}_{26} \mathrm{H}_{24} \mathrm{Cl}_{2} \mathrm{~N}_{2} \mathrm{O}_{3} .0 .5 \mathrm{H} \mathrm{H}_{2} \mathrm{O}$ C, 63.4 ; H, 5.1; N, 5.7. Found: C, 63.6; H. 4.8; N, 5.5\%.

Methyl 3-(4-chlorophenyl)-4,6-dimethoxy-2-( $N, N^{\prime}$-dimethylaminomethyl)-indole-7-carboxylate (23). This was prepared according to the method of preparation of compound 22 using indole 21 (70 mg, $0.20 \mathrm{mmol}$ ) and produced compound $23(61 \mathrm{mg}, 75 \%)$ as a white solid, $\mathrm{mp} 138{ }^{\circ} \mathrm{C}$ (from MeOH). IR $\left(\mathrm{v}_{\max }, \mathrm{cm}^{-1}\right): 3420$, 1660, 1580, 1370, 1300, 1260, 1185, 1165. UV/Vis $\left(\lambda_{\max }, \mathrm{nm}, \varepsilon, \mathrm{cm}^{-1} \mathrm{M}^{-1}\right): 240(38,200), 223(27,600), 215$ (25,900), 306 (17,800). ${ }^{1} \mathrm{H}$ NMR (300 MHz, CDCl $): \delta_{\mathrm{H}} 2.12(6 \mathrm{H}, \mathrm{s}, \mathrm{Me}), 3.47\left(2 \mathrm{H}, \mathrm{s}, \mathrm{CH}_{2}\right), 3.78(3 \mathrm{H}, \mathrm{s}, \mathrm{OMe}), 3.97$ $(6 \mathrm{H}, \mathrm{s}, \mathrm{OMe}), 6.22(1 \mathrm{H}, \mathrm{s}, \mathrm{H} 5), 7.31(4 \mathrm{H}, \mathrm{s}, \mathrm{aryl}), 10.27(1 \mathrm{H}, \mathrm{br} \mathrm{s}, \mathrm{NH}) .{ }^{13} \mathrm{C} \mathrm{NMR}(75 \mathrm{MHz}, \mathrm{CDCl}): \delta_{\mathrm{C}} 45.4(\mathrm{Me})$, $51.7\left(\mathrm{CH}_{2}\right.$ ), 54.6, 55.2, 57.5 (OMe), 88.8 (C5), 127.3, 132.3 (aryl CH), 95.5, 110.9, 114.0, 131.8, 132.8, 133.9, 138.1, 158.4, 159.8 (aryl C), 167.7 (C=O). MS (+EI, $m / z, \%): 403(M+1,50), 350$ (100). Anal. calcd for $\mathrm{C}_{21} \mathrm{H}_{23} \mathrm{ClN}_{2} \mathrm{O}_{4}$. 0.3 $\mathrm{H}_{2} \mathrm{O}: \mathrm{C}, 61.8 ; \mathrm{H}, 5.8 ; \mathrm{N}, 6.9$. Found: $\mathrm{C}, 61.5 ; \mathrm{H}, 6.0 ; \mathrm{N}, 7.1 \%$. 
$\mathbf{N}, \mathbf{N}$-Di-(3-(4-chlorophenyl)-4,6-dimethoxy-7-trichloroacetyl)-2-indolylmethyl) benzylamine (24). A mixture of indole 20 (1.0 g, $2.3 \mathrm{mmol})$, and compound $12(0.22 \mathrm{~g}, 1.15 \mathrm{mmol})$ in $\mathrm{CH}_{2} \mathrm{Cl}_{2}$ (15 ml) was treated with trichloromethylsilane $(0.30 \mathrm{~mL})$. The mixture was stirred at $\mathrm{rt}$ for $2 \mathrm{~d}$, water $(15 \mathrm{~mL})$ was added and the organic layer was separated and dried $\left(\mathrm{MgSO}_{4}\right)$. The solvent was evaporated off and the residue purfied by chromatographey using $\mathrm{CH}_{2} \mathrm{Cl}_{2}$-light petroleum (1:1) as eluent to give compound 24 (0.75 g, 64\%) as a yellow solid, $\mathrm{mp} 186^{\circ} \mathrm{C}\left(\mathrm{CH}_{2} \mathrm{Cl}_{2}\right.$-light petroleum). IR $\left(v_{\max }, \mathrm{cm}^{-1}\right): 3450,1610,1570,1490,1200,1150,980,790$. UV/Vis $\left(\lambda_{\max }, \mathrm{nm}, \varepsilon, \mathrm{cm}^{-1} \mathrm{M}^{-1}\right): 224$ (42,900), 260 (33,500), 345 (18,600). ${ }^{1} \mathrm{H} \mathrm{NMR}\left(300 \mathrm{MHz}, \mathrm{CDCl}_{3}\right): \delta_{\mathrm{H}} 3.44\left(2 \mathrm{H}, \mathrm{s}, \mathrm{CH}_{2}\right)$, $3.59\left(4 \mathrm{H}, \mathrm{s}, \mathrm{CH}_{2}\right), 3.79,3.94$ (each $\left.6 \mathrm{H}, 2 \mathrm{~s}, \mathrm{OMe}\right), 6.12(2 \mathrm{H}, \mathrm{s}, \mathrm{H} 5), 7.15-7.22(8 \mathrm{H}, \mathrm{m}$, aryl), 7.28-7.31 (5H, m, aryl), $10.23\left(2 \mathrm{H}\right.$, br s, NH). ${ }^{13} \mathrm{C} \mathrm{NMR}\left(75 \mathrm{MHz}, \mathrm{CDCl}_{3}\right): \delta_{\mathrm{C}} 49.5,58.6\left(\mathrm{CH}_{2}\right), 55.3,55.6(\mathrm{OMe}), 87.6$ (C5), 127.5 , 128.6, 128.9, 131.4, 132.1 (aryl CH), $98.5\left(\mathrm{CCl}_{3}\right), 98.4,112.1,115.6,127.3,133.3,137.9,138.4,159.8,160.7$ (aryl C), $181.7(\mathrm{C}=0)$. MS (+El, m/z, \%): $1000(\mathrm{M}+3,15) .553$ (15), 446 (30), 356 (100). Anal. calcd for $\mathrm{C}_{45} \mathrm{H}_{35} \mathrm{Cl}_{8} \mathrm{~N}_{3} \mathrm{O}_{6}$. $0.6 \mathrm{CH}_{2} \mathrm{Cl}_{2}$ : C, 52.2; $\mathrm{H}, 3.5 ; \mathrm{N}, 4.0$. Found: $\mathrm{C}, 52.2 ; \mathrm{H}, 3.5 ; \mathrm{N}, 4.0 \%$.

A similar reaction in which the trichloromethylsilane was replaced by trifluoroacetic anhydride $(0.1 \mathrm{~mL}, 0.2$ $\mathrm{mmol}$ ) gave compound 24 (0.70 g, 60\%).

N,N-Di-(3-(4-chlorophenyl)-7-formyl-4,6-dimethoxy-2-indolylmethyl)benzylamine (26). This was prepared according to the method of preparation of compound 24 using indole 25 (0.91 g, 2.9 mmol), compound 12 $(0.30 \mathrm{~g}, 1.5 \mathrm{mmol})$ and trichloromethylsilane $(0.30 \mathrm{~mL})$. Chromatography using EtOAc-light petroleum (1:1) as eluent gave compound $26(0.88 \mathrm{~g}, 80 \%)$ as a yellow solid, $\mathrm{mp} 248{ }^{\circ} \mathrm{C}$ (from $\mathrm{CH}_{2} \mathrm{Cl}_{2}$-light petroleum). IR ( $V_{\max }$, $\left.\mathrm{cm}^{-1}\right)$ : 3400, 1620, 1580, 1240, 1210, 1180, 1090, 990, 790, 720. UV/Vis $\left(\lambda_{\max }, \mathrm{nm}, \varepsilon, \mathrm{cm}^{-1} \mathrm{M}^{-1}\right): 255(42,800)$, 324 (24,400). ${ }^{1} \mathrm{H}$ NMR (300 MHz, CDCl 3$): \delta_{\mathrm{H}} 3.42\left(2 \mathrm{H}, \mathrm{s}, \mathrm{CH}_{2}\right), 3.58\left(4 \mathrm{H}, \mathrm{s}, \mathrm{CH}_{2}\right), 3.80,3.96$ (each 6H, 2s, OMe), $6.11(2 \mathrm{H}, \mathrm{s}, \mathrm{H} 5), 7.19-7.29(13 \mathrm{H}, \mathrm{m}, \mathrm{aryl}), 10.38(2 \mathrm{H}, \mathrm{s}, \mathrm{CHO}), 10.58(2 \mathrm{H}, \mathrm{br} \mathrm{s}, \mathrm{NH}) .{ }^{13} \mathrm{C} \mathrm{NMR}\left(75 \mathrm{MHz}, \mathrm{CDCl}_{3}\right): \delta_{\mathrm{c}}$ 49.1, $58.2\left(\mathrm{CH}_{2}\right), 55.3,56.5$ (OMe), 86.8 (C5), 127.5, 128.6, 128.9, 131.9, 132.5 (aryl CH), 104.4, 111.5, 114.8, 127.3, 132.0, 133.5, 136.4, 138.0, 160.5, 162.6 (aryl C), 188.0 (C=O). MS (+EI, m/z, \%): 762 (M, 30$), 279$ (50), 263 (55), 203 (100). Anal. calcd for $\mathrm{C}_{43} \mathrm{H}_{37} \mathrm{Cl}_{2} \mathrm{~N}_{3} \mathrm{O}_{6} .0 .8 \mathrm{CH}_{2} \mathrm{Cl}_{2}: \mathrm{C}, 63.3 ; \mathrm{H}, 4.7 ; \mathrm{N}, 5.1$. Found: $\mathrm{C}, 63.2 ; \mathrm{H}, 4.9 ; \mathrm{N}$, $4.7 \%$.

Dimethyl di-[2-(3-(4-chlorophenyl)-4,6-dimethoxyindolyl)]methane-7,7'-dicarboxylate (27). To the mixture of indole 21 (0.13 g, $0.37 \mathrm{mmol}$ ) and formaldehyde solution (40\%, $1.6 \mathrm{~mL}, 21.6 \mathrm{mmol}$ ) in MeOH (20 mL) was added conc. $\mathrm{HCl}(1 \mathrm{~mL})$. The mixture was heated under reflux for $3 \mathrm{~h}$. The mixture was allowed to cool to rt, the resulting precipitate was filtered off, washed with water and dried to afford compound 27 (73 mg, $56 \%$ ) as a yellow solid, $\mathrm{mp} 198{ }^{\circ} \mathrm{C}$ (from MeOH-CH${ }_{2} \mathrm{Cl}_{2}$ ). IR ( $\left.v_{\max }, \mathrm{cm}^{-1}\right)$ : 3330, 1650, 1580, 1540, 1140, 1080, $980,790$. UV/Vis $\left(\lambda_{\max }, \mathrm{nm}, \varepsilon, \mathrm{cm}^{-1} \mathrm{M}^{-1}\right): 243$ (23,000), $308(18,400) .{ }^{1} \mathrm{H}$ NMR $\left.\left(300 \mathrm{MHz}^{\mathrm{CDCl}}\right)_{3}\right): \delta_{\mathrm{H}} 3.77,3.87,3.94$ (each $6 \mathrm{H}, 3 \mathrm{~s}, \mathrm{OMe}), 4.09\left(2 \mathrm{H}, \mathrm{s}, \mathrm{CH}_{2}\right), 6.24(2 \mathrm{H}, \mathrm{s}, \mathrm{H} 5), 7.30(8 \mathrm{H}, \mathrm{s}, \operatorname{aryl}), 9.83(2 \mathrm{H}, \mathrm{br} \mathrm{s}, \mathrm{NH}) .{ }^{13} \mathrm{C} \mathrm{NMR}\left(75 \mathrm{MHz}^{\mathrm{CDCl}} \mathrm{CD}_{3}\right):$ $\delta_{c} 23.5\left(\mathrm{CH}_{2}\right), 51.6,55.2,57.4$ (OMe), 89.1 (C5), 127.6, 132.1 (aryl CH), 95.4, 112.0, 113.9, 130.4, 130.8, 133.6, 138.2, 158.4, 159.9 (aryl C), 167.4 (C=O). Anal. calcd for $\mathrm{C}_{37} \mathrm{H}_{32} \mathrm{Cl}_{2} \mathrm{~N}_{2} \mathrm{O}_{8} .0 .5 \mathrm{H}_{2} \mathrm{O}: \mathrm{C}, 62.4 ; \mathrm{H}, 4.7 ; \mathrm{N}, 3.9$. Found: C, 62.0; H, 4.6; N, 4.0\%.

Di-[2-(3-(4-chlorophenyl)-7-(4-chlorobenzoyl)-4,6-dimethoxyindolyl]methane (29). This was prepared according to the method of preparation of compound 27 from indole 19 (0.3 g, $0.7 \mathrm{mmol})$, formaldehyde solution (40\%, $3.2 \mathrm{~mL}, 43.2 \mathrm{mmol})$ in $\mathrm{MeOH}(40 \mathrm{~mL})$ with added conc. $\mathrm{HCl}(2 \mathrm{~mL})$ and gave compound $29(210$ $\mathrm{mg}, 70 \%)$ as a yellow solid, $\mathrm{mp} 234^{\circ} \mathrm{C}$. IR $\left(v_{\max }, \mathrm{cm}^{-1}\right): 3400,1640,1580,1540,1260,1210,1190,900$. UV/Vis $\left(\lambda_{\max }, \mathrm{nm}, \varepsilon, \mathrm{cm}^{-1} \mathrm{M}^{-1}\right): 205$ (74,300), 260 (42,700), 332 (17,400). ${ }^{1} \mathrm{H} \mathrm{NMR}\left(300 \mathrm{MHz} \mathrm{CDCl}_{3}\right): \delta_{\mathrm{H}} 3.57,3.78$ (each $6 \mathrm{H}, 2 \mathrm{~s}, \mathrm{OMe}), 4.11\left(2 \mathrm{H}, \mathrm{s}, \mathrm{CH}_{2}\right), 6.12(2 \mathrm{H}, \mathrm{s}, \mathrm{H} 5), 7.32-7.34(12 \mathrm{H}, \mathrm{m}, \operatorname{aryl}), 7.49(4 \mathrm{H}, \mathrm{d}, \mathrm{J} 8.7 \mathrm{~Hz}, \operatorname{aryl}), 9.85(2 \mathrm{H}$, br s, NH). ${ }^{13} \mathrm{C} \mathrm{NMR} \mathrm{(75} \mathrm{MHz,} \mathrm{CDCl}_{3}$ ): $\delta_{\mathrm{C}} 23.4\left(\mathrm{CH}_{2}\right.$ ), 55.3, 56.2 (OMe), 88.1 (C5), 127.7, 127.8, 129.6, 132.2 (aryl $\mathrm{CH}), 103.7,112.3,114.4,130.5,133.5,136.6,138.0,140.8,159.4,159.6$ (aryl C), 194.4 (C=O). Anal. calcd for $\mathrm{C}_{47} \mathrm{H}_{34} \mathrm{Cl}_{4} \mathrm{~N}_{2} \mathrm{O}_{6} .1 .0 \mathrm{H}_{2} \mathrm{O}: \mathrm{C}, 64.0 ; \mathrm{H}, 4.1 ; \mathrm{N}, 3.2$. Found: $\mathrm{C}, 63.9 ; \mathrm{H}, 3.9 ; \mathrm{N}, 3.1 \%$. 
Di-[2-(3-(4-chlorophenyl)-4,6-dimethoxy-7-trichloroacetyl)indolyl]methane (30). This was prepared according to the method of preparation of compound 27 from indole 20 ( $0.1 \mathrm{~g}, 0.23 \mathrm{mmol})$, formaldehyde solution (40\%, $1.0 \mathrm{~mL}, 13.5 \mathrm{mmol})$ in $\mathrm{MeOH}(20 \mathrm{~mL})$ with added conc. $\mathrm{HCl}(1 \mathrm{~mL})$ and after heating for $2 \mathrm{~d}$ gave compound 30 (90 mg, 68\%) as a yellow solid, $\mathrm{mp}>330{ }^{\circ} \mathrm{C}$ (from $\left.\mathrm{CH}_{2} \mathrm{Cl}_{2}-\mathrm{MeOH}\right)$. IR $\left(v_{\max }, \mathrm{cm}^{-1}\right): 3400,1630$, 1580, 1370, 1240, 1220, 1160, 985. UV/Vis $\left(\lambda_{\max }, \mathrm{nm}, \varepsilon, \mathrm{cm}^{-1} \mathrm{M}^{-1}\right): 262(56,100), 343(43,100) .{ }^{1} \mathrm{H} N \mathrm{NMR}(300$ $\mathrm{MHz}_{\mathrm{CDCl}}$ ): $\delta_{\mathrm{H}} 3.81,3.94$ (each $\left.6 \mathrm{H}, 2 \mathrm{~s}, \mathrm{OMe}\right), 4.08\left(2 \mathrm{H}, \mathrm{s}, \mathrm{CH}_{2}\right), 6.14(2 \mathrm{H}, \mathrm{s}, \mathrm{H} 5), 7.34(8 \mathrm{H}, \mathrm{s}, \operatorname{aryl}), 9.84(2 \mathrm{H}, \mathrm{br}$ s, NH). ${ }^{13} \mathrm{C} \mathrm{NMR}\left(75 \mathrm{MHz}, \mathrm{CDCl}_{3}\right): \delta_{\mathrm{C}} 23.3\left(\mathrm{CH}_{2}\right), 55.4,55.6$ (OMe), 87.7 (C5), 127.8, $132.1(\operatorname{aryl~CH}), 98.4\left(\mathrm{CCl}_{3}\right)$, $112.3,114.9,130.1,131.9,132.5,133.1,138.6,160.0,160.9$ (aryl C), 182.0 (C=0). Anal. calcd for $\mathrm{C}_{37} \mathrm{H}_{26} \mathrm{Cl}_{8} \mathrm{~N}_{2} \mathrm{O}_{6}$. $2.5 \mathrm{H}_{2} \mathrm{O}: \mathrm{C}, 48.1 ; \mathrm{H}, 3.4 ; \mathrm{N}, 3.0$. Found: $\mathrm{C}, 48.0 ; \mathrm{H}, 3.0 ; \mathrm{N}, 3.0 \%$.

Di-[2-(3-(4-chlorophenyl)-4,6-dimethoxyindolyl]methane-7,7'-dicarboxamide (31). The diindolylmethane 30 $(0.50 \mathrm{~g}, 0.57 \mathrm{mmol})$ was partiallly dissolved in acetonitrile $(10 \mathrm{~mL})$ and concentrated ammonia was added until the mixture was basic. The mixture was stirred at $\mathrm{rt}$ for $1 \mathrm{~h}$. The resulting white precipitate was filtered, washed with acetonitrile and dried to yield compound $31(0.14 \mathrm{~g}, 36 \%), \mathrm{mp} 310{ }^{\circ} \mathrm{C} . \mathrm{IR}\left(\mathrm{V}_{\max }, \mathrm{cm}^{-1}\right): 3420,3380$, 3320, 1630, 1600, 1560, 1545, 1220, 1200, 1100. UV/Vis $\left(\lambda_{\max }, \mathrm{nm}, \varepsilon, \mathrm{cm}^{-1} \mathrm{M}^{-1}\right): 207(6,500) 304(2,700) .{ }^{1} \mathrm{H}$ NMR $\left(300 \mathrm{MHz}, \mathrm{CDCl}_{3}\right): \delta_{\mathrm{H}}$ 3.76, 3.98 (each 6H, 2s, OMe), $4.10\left(2 \mathrm{H}, \mathrm{s}, \mathrm{CH}_{2}\right), 5.55(2 \mathrm{H}, \mathrm{br} \mathrm{s}, \mathrm{NH}), 6.18(2 \mathrm{H}, \mathrm{s}$, $\mathrm{H} 5), 7.29(8 \mathrm{H}, \mathrm{s}, \mathrm{aryl}), 7.78(2 \mathrm{H}, \mathrm{br} \mathrm{s}, \mathrm{NH}), 10.72(2 \mathrm{H}, \mathrm{br} \mathrm{s}, \mathrm{NH}) .{ }^{13} \mathrm{C} \mathrm{NMR}\left(75 \mathrm{MHz}, \mathrm{CDCl}_{3}\right): \delta_{\mathrm{C}} 23.5\left(\mathrm{CH}_{2}\right), 55.2$, 56.9 (OMe), 87.9 (C5), 127.5, 132.3 (aryl CH), 96.9, 113.2, 160.7, 131.1, 131.9, 133.8, 138.4, 156.9, 157.3 (aryl C), 169.3 (C=O). Anal. calcd for $\mathrm{C}_{35} \mathrm{H}_{30} \mathrm{Cl}_{2} \mathrm{~N}_{4} \mathrm{O}_{6}$. $1.5 \mathrm{H}_{2} \mathrm{O}: \mathrm{C}, 60.0 ; \mathrm{H}, 4.7 ; \mathrm{N}, 8.0$. Found: $\mathrm{C}, 60.2 ; \mathrm{H}, 4.4 ; \mathrm{N}, 8.0 \%$.

2,2'-Di-[3-(4-chlorophenyl)-4,6-dimethoxyindolyl]methane-7,7'-dicarboxylic acid (32). A mixture of diindolylmethane 27 (0.55 g, $0.62 \mathrm{mmol})$ in $\mathrm{EtOH}(20 \mathrm{~mL})$ containing $\mathrm{KOH}(1.50 \mathrm{~g}, 26 \mathrm{mmol})$ was heated under reflux for $2 \mathrm{~h}$. The mixture was allowed to cool to $\mathrm{rt}$ and acidified with $\mathrm{HCl}(0.1 \mathrm{M})$. The resulting precipitate was filtered off, washed with water and dried to yield compound $32(0.40 \mathrm{~g}, 75 \%), \mathrm{mp} 277{ }^{\circ} \mathrm{C}\left(\mathrm{from} \mathrm{CH}_{2} \mathrm{Cl}_{2-}\right.$ $\mathrm{MeOH})$. IR $\left(v_{\max }, \mathrm{cm}^{-1}\right): 3500-3100,1670,1580,1190,980$. UV/Vis $\left(\lambda_{\max }, \mathrm{nm}, \varepsilon, \mathrm{cm}^{-1} \mathrm{M}^{-1}\right): 249(43,700), 312$ (37,000). ${ }^{1} \mathrm{H}$ NMR $\left(300 \mathrm{MHz}, \mathrm{CDCl}_{3}\right): \delta_{\mathrm{H}} 3.78,4.08$ (each $\left.6 \mathrm{H}, 2 \mathrm{~s}, \mathrm{OMe}\right), 4.12\left(2 \mathrm{H}, \mathrm{s}, \mathrm{CH}_{2}\right), 6.19(2 \mathrm{H}, \mathrm{s}, \mathrm{H} 5), 7.32$ $(8 \mathrm{H}, \mathrm{s}, \operatorname{aryl}), 10.53(2 \mathrm{H}, \mathrm{b}, \mathrm{NH}), 10.72\left(2 \mathrm{H}, \mathrm{br}\right.$ s, COOH). ${ }^{13} \mathrm{C} \mathrm{NMR}\left(75 \mathrm{MHz}, \mathrm{CDCl}_{3}\right): \delta_{\mathrm{c}} 23.5\left(\mathrm{CH}_{2}\right), 55.4,57.5$ (OMe), 87.4 (C5), 127.7, 132.2 (aryl CH), 92.3, 94.0, 112.8, 114.1, 132.2, 133.2, 137.8, 157.4, 158.7 (aryl C), 168.8 (C=O). Anal. calcd for $\mathrm{C}_{35} \mathrm{H}_{28} \mathrm{Cl}_{2} \mathrm{~N}_{2} \mathrm{O}_{8}$. $3 \mathrm{H}_{2} \mathrm{O}: \mathrm{C}, 57.6 ; \mathrm{H}, 4.7 ; \mathrm{N}, 3.8$. Found: $\mathrm{C}, 57.4 ; \mathrm{H}, 4.3 ; \mathrm{N}, 3.9 \%$.

\section{3-(N-Benzy1)-6,27-di-(4-chlorophenyl)-8,10,23,25-tetramethoxy-3,13,20,28,31-pentaazahexacyclo-} $\left[20.5 \cdot 2 \cdot 2^{5,11} \cdot 0^{7,30} \cdot 0^{14,19} \cdot 0^{26,29}\right]$ hentriaconta-5,7,9,11(30),12, 14,16,18,20,22,24,26(29),27(21)tridecene (33). The mixture of indole $28(0.12 \mathrm{~g}, 0.2 \mathrm{mmol})$ and 1,2-diaminobenzene $(17.5 \mathrm{mg}, 0.2 \mathrm{mmol})$ in dry toluene (20 $\mathrm{mL}$ ) containing activated 4A molecular sieves $(5 \mathrm{~g})$ was heated under reflux for $2 \mathrm{~d}$ under a $\mathrm{N}_{2}$ atmosphere. The solvent was evaporated off and the residue purified by chromatography with $\mathrm{CH}_{2} \mathrm{Cl}_{2}$-light petroleum (3:2) as eluent to give compound 33 (40 mg, 40\%) as a yellow solid, $\mathrm{mp}>330{ }^{\circ} \mathrm{C}$ (from $\mathrm{CH}_{2} \mathrm{Cl}_{2}$-light petroleum). IR $\left(v_{\max }, \mathrm{cm}^{-1}\right): 3330,1580,1550,1500,1240,1200,1150,1100,990,790$. UV/Vis $\left(\lambda_{\max }, \mathrm{nm}, \varepsilon, \mathrm{cm}^{-1} \mathrm{M}^{-1}\right): 236$ (67,000), 362 (24,100). ${ }^{1} \mathrm{H}$ NMR (300 MHz, CDCl 3$): \delta_{\mathrm{H}} 3.56\left(2 \mathrm{H}, \mathrm{s}, \mathrm{CH}_{2}\right), 3.62\left(4 \mathrm{H}, \mathrm{s}, \mathrm{CH}_{2}\right), 3.78,3.94($ each $6 \mathrm{H}$, 2s, OMe), $6.19(2 \mathrm{H}, \mathrm{s}, \mathrm{H} 9, \mathrm{H} 24), 6.90(2 \mathrm{H}, \mathrm{t}, J 7.5 \mathrm{~Hz}, \operatorname{aryl}), 7.00(1 \mathrm{H}, \mathrm{t}, J 7.7 \mathrm{~Hz}, \operatorname{aryl}), 7.18-7.34(12 \mathrm{H}, \mathrm{m}, \operatorname{aryl})$, $7.47\left(2 \mathrm{H}, \mathrm{d}, J 7.7 \mathrm{~Hz}\right.$, aryl), $9.10(2 \mathrm{H}, \mathrm{s}, \mathrm{CH}=\mathrm{N}), 12.01(2 \mathrm{H}, \mathrm{br} \mathrm{s}, \mathrm{NH}) .{ }^{13} \mathrm{C} \mathrm{NMR}\left(75 \mathrm{MHz}, \mathrm{CDCl}_{3}\right): \delta_{\mathrm{C}} 48.7,58.0$ $\left(\mathrm{CH}_{2}\right), 55.2,56.9$ (OMe), 87.6 (C9, C24), 126.2, 127.2, 127.4, 128.3, 129.1, 132.1, 132.5 (aryl CH), 102.8, 111.6, 114.1, 119.1, 131.6, 133.8, 136.3, 138.8, 147.0, 157.7, 158.9 (aryl C), 155.6 (CH=N). MS (MALDI, m/z, \%): 834 (M, 15), 742 (40), 715 (100). 417 (60). Anal. calcd for $\mathrm{C}_{49} \mathrm{H}_{41} \mathrm{Cl}_{2} \mathrm{~N}_{5} \mathrm{O}_{4}$. 0.5 $\mathrm{H}_{2} \mathrm{O}: \mathrm{C}, 69.7 ; \mathrm{H}, 5.0 ; \mathrm{N}$, 8.3. Found: C, $69.5 ; H, 5.1 ; N, 8.1 \%$.

\section{3-(N-Benzyl)-6,23-di-(4-chlorophenyl)-8,10,19,21-tetramethoxy-3,13,16,24,27-pentaazapentacyclo-}

$\left[17.5 \cdot 2 \cdot 2^{5,11} \cdot 0^{17,26} \cdot 0^{22,25}\right]$ heptacosa-5,7,9,11(26),16,19,21,23(1), 25(18)decene (34). This was prepared according to the method of preparation of compound 33 using indole 28 (0.10 g, $0.13 \mathrm{mmol})$ and 1,2- 
diaminoethane $(8.0 \mathrm{mg}, 0.13 \mathrm{mmol})$. After heating the reaction mixture in toluene $(20 \mathrm{~mL})$ under reflux overnight, the solvent was evaporated off and the residue purified by chromatography using EtOAc-light petroleum (1:1) as eluent to afford compound 34 (30 mg, 30\%) as a yellow solid, mp $264{ }^{\circ} \mathrm{C}$ (from $\mathrm{CH}_{2} \mathrm{Cl}_{2}-$ light petroleum). IR ( $\left.v_{\max }, \mathrm{cm}^{-1}\right)$ : 3320, 1580, 1330, 1240, 1210, 1180, 1150, 980, 900. UV/Vis $\left(\lambda_{\max }, \mathrm{nm} \varepsilon, \mathrm{cm}^{-1} \mathrm{M}^{-1}\right)$ : 339 (24,200). ${ }^{1} \mathrm{H}$ NMR $\left(300 \mathrm{MHz}_{,} \mathrm{CDCl}_{3}\right): \delta_{\mathrm{H}} 3.37\left(2 \mathrm{H}, \mathrm{s}, \mathrm{CH}_{2}\right), 3.64,4.07$ (each $\left.4 \mathrm{H}, 2 \mathrm{~s}, \mathrm{CH}_{2}\right), 3.77,3.94$ (each $6 \mathrm{H}$, 2s, OMe), $6.19(2 \mathrm{H}, \mathrm{s}, \mathrm{H} 5), 7.12-7.20(5 \mathrm{H}, \mathrm{m}, \operatorname{aryl}), 7.25-7.30(7 \mathrm{H}, \mathrm{m}, \operatorname{aryl}), 7.33(1 \mathrm{H}, \mathrm{s}, \operatorname{aryl}), 9.00(2 \mathrm{H}, \mathrm{s}, \mathrm{CH}=\mathrm{N})$, $11.84(2 \mathrm{H}, \mathrm{br} \mathrm{s}, \mathrm{NH}) .{ }^{13} \mathrm{C} \mathrm{NMR}\left(75 \mathrm{MHz}_{\mathrm{CDCl}}\right): \delta_{\mathrm{c}} 49.2,56.8,63.3\left(\mathrm{CH}_{2}\right), 55.2,57.0$ (OMe), 87.7 (C5), 127.4, 128.1, 129.2, 132.2, 132.4 (aryl CH), 101.9, 111.6, 114.0, 127.0, 131.6, 134.1, 136.4, 137.9, 156.7, 157.6 (aryl C), $158.1(\mathrm{CH}=\mathrm{N})$. MS (ES, $\mathrm{m} / z, \%): 786(\mathrm{M}, 10), 403(90), 328(100)$. Anal. calcd for $\mathrm{C}_{45} \mathrm{H}_{41} \mathrm{Cl}_{2} \mathrm{~N}_{5} \mathrm{O}_{4} .1 .6 \mathrm{CH}_{2} \mathrm{Cl}_{2}: \mathrm{C}_{\text {, }}$ 60.7; H, 4.8; N, 7.6. Found: C, 60.9; H, 5.0; N, 7.1\%.

\section{3-(N-Benzyl)-6,27-di-(4-chlorophenyl)-8,10,23,25-tetramethoxy-3,13,20,28,31-pentaazahexacyclo-} $\left[20.5 \cdot 2 \cdot 2^{5,11} \cdot 0^{7,30} \cdot 0^{14,19} \cdot 0^{26,29}\right]$ hentriaconta-5,7,9,11(30),12, 14,16,18,20,22,24,26(29),27(21)tridecenato-2nickel(II) (35). Nickel (II) acetate tetrahydrate (19.4 mg, $0.1 \mathrm{mmol})$ as a suspension in acetonitrile (3 $\mathrm{mL}) \mathrm{was}$ added to the macrocycle $33(65 \mathrm{mg}, 0.1 \mathrm{mmol})$ in acetonitrile $(75 \mathrm{~mL})$ containing triethylamine $(4 \mathrm{drops})$ at reflux. The mixture was heated under reflux overnight and cooled to rt. The resulting precipitate was filtered off, washed with acetonitrile and dried to yield compound 35 (60 mg, 86\%) as a brown solid, $\mathrm{mp} 312{ }^{\circ} \mathrm{C}$ (from $\mathrm{CH}_{2} \mathrm{Cl}_{2}$-light petroleum). IR ( $\left.v_{\max }, \mathrm{cm}^{-1}\right): 1600-1500,1340,1300,1270,1160,1080,990$. UV/Vis $\left(\lambda_{\max }, \mathrm{nm}, \varepsilon, \mathrm{cm}^{-}\right.$ $\left.{ }^{1} \mathrm{M}^{-1}\right): 209$ (315,000), 296 (11,470), 466 (503). ${ }^{1} \mathrm{H}$ NMR (300 MHz, CDCl $): \delta_{\mathrm{H}} 2.68\left(2 \mathrm{H}, \mathrm{s}, \mathrm{CH}_{2}\right), 3.37\left(4 \mathrm{H}, \mathrm{s}, \mathrm{CH}_{2}\right)$, 3.80, 4.01 (each 6H, 2s, OMe), $6.09(2 \mathrm{H}, \mathrm{s}, \mathrm{H} 5), 6.64(2 \mathrm{H}, \mathrm{br} \mathrm{s}, \operatorname{aryl}), 7.06-7.42(13 \mathrm{H}, \mathrm{m}, \operatorname{aryl}), 7.42(2 \mathrm{H}, \mathrm{br} \mathrm{s}$, aryl), $8.41(2 \mathrm{H}, \mathrm{s}, \mathrm{CH}=\mathrm{N}) .{ }^{13} \mathrm{C} \mathrm{NMR}\left(75 \mathrm{MHz}, \mathrm{CDCl}_{3}\right): \delta_{\mathrm{c}} 47.5,57.6\left(\mathrm{CH}_{2}\right), 55.3,56.5$ (OMe), 85.7 (C5), 126.1 , 126.2, 127.2, 127.8, 128.3, 130.9, 132.6 (aryl CH), 105.9, 113.6, 116.5, 119.1, 136.3, 139.1, 139.6, 143.6, 145.6, 161.2, 161.5 (aryl C), $148.2(\mathrm{C}=\mathrm{N})$. MS (MALDI, $\mathrm{m} / z, \%): 892(\mathrm{M}+1,20)$. Anal. calcd for $\mathrm{C}_{49} \mathrm{H}_{39} \mathrm{Cl}_{2} \mathrm{~N}_{5} \mathrm{O}_{4} \mathrm{Ni}_{0} 0.5$ $\mathrm{H}_{2} \mathrm{O}: \mathrm{C}, 65.4 ; \mathrm{H}, 4.5 ; \mathrm{N}, 7.8$. Found: $\mathrm{C}, 65.1 ; \mathrm{H}, 4.3 ; \mathrm{N}, 7.8 \%$.

\section{Acknowledgements}

Financial support from the Australian Research Council is gratefully acknowledged. B.P. also acknowledges receipt of an International Postgraduate Research Scholarship from the Australian Government.

\section{References}

1. Brehm, W. J.; Lindwall, H. G. J. Org. Chem. 1950, 15, 685-687. https://doi.org/10.1021/jo01149a039

2. Joule, J. A. Science of Synthesis 2001, 10, 361-652.

3. Brown, V. H.; Skinner, W. A.; De Graw, J. I. J. Heterocycl. Chem. 1969, 6, 539-543. https://doi.org/10.1002/jhet.5570060414

4. Crohare, R.; Merkuza, V. M.; Gonzalez, H. A.; Ruveda, E. A. J. Heterocycl. Chem. 1970, 7, 729-732. https://doi.org/10.1002/jhet.5570070349

5. Condie, G. C.; Channon, M. F.; Ivory, A. J.; Kumar, N.; Black, D. StC. Tetrahedron 2005, 61, 4989-5004. https://doi.org/10.1016/j.tet.2005.03.048

6. Black, D. StC.; Bowyer, M. C.; Catalano, M. M.; Ivory, A. J.; Keller, P. A.; Kumar, N.; Nugent, S. J. Tetrahedron 1994, 50, 10497-10508. 
https://doi.org/10.1016/S0040-4020(01)89590-9

7. Tramontini, M.; Angiolini, L. Tetrahedron 1990, 46, 1791-1837.

https://doi.org/10.1016/S0040-4020(01)89752-0

8. Earle, M. J.; Fairhurst, R. A.; Heaney, H.; Papageorgiou, G.; Wilkins, R. F. Tetrahedron Lett. 1990, 31, 42294232.

https://doi.org/10.1016/S0040-4039(00)97589-0

9. Heaney, H.; Papageorgiou, G. Tetrahedron 1996, 52, 3473-3486.

https://doi.org/10.1016/0040-4020(96)00026-9

10. Buckley, B. R.; Boxhall, J. Y.; Bulman Page, P. C.; Chan, Y.; Elsegood, M. R. J.; Heaney, H.; Holmes, K. E.; Mclldowie, M. J.; McKee, V.; McGrath, M. J.; Mocerino, M.; Poulton, A. M.; Sampler, E. P.; Skelton, B. W.; White, A. H. Eur. J. Org. Chem. 2006, 5117-5134.

https://doi.org/10.1002/ejoc.200600590

11. Brocke, C. ; Brimble, M. A. ; Lin, D. S.-H. ; McLeod, M. D. Synlett, 2004, 2359-2363.

12. Shi, Y. ; Wang, Q. ; Gao, S. Org. Chem. Front. 2018, 5, 1049-1066.

https://doi.org/10.1039/C7Q001079F

13. Purwono, B.; Kumar, N.; Black, D. StC. Arkivoc, 2022, 4, 6-23.

https://doi.org/10.24820/ark.5550190.p011.637

14. Bowyer, P. K.; Black, D. StC.; Craig, D. C.; Rae, A. D.; Willis, A. C. J. Chem. Soc., Dalton Trans. 2001, 19481958.

https://doi.org/10.1039/C7Q001079F

15. Bowyer, P. K.; Black, D. StC.; Craig, D. C. Tetrahedron 2005, 61, 10781-10792.

https://doi.org/10.1016/j.tet.2005.08.075

16. Kandemir, H. ; Sengul, I. F. ; Kumar, N. ; Black, D. StC. Aust. J. Chem. 2017, 70, 1196-1201.

https://doi.org/10.1071/CH17264

17. Somphol, K.; Kumar, N.; Black, D. StC. Heterocycles, 2021, 102, 686-704.

https://doi.org/10.3987/COM-21-14411 